\title{
Research on the Transformation Strategies of the natural Lighting in the Traditional Silt Houses in Yunnan Province
}

\author{
Zhou Zhiyong $^{1}$ Zhuchi $^{1}$ DuanZhuowei $^{2}$
}

1 Kunming University of Science and Technology, Architecture and Civil Engineering, China.

2 Kunming architectural design and research institute co.,LTD,China.

657434674@qq.com,547812209@qq.com,13085397335@126.com

Keywords: Stilt houses; Natural lighting; Daylight simulation; transformation strategies

Abstract: In this paper, the traditional silt house of Wa ethnicity in Yunnan province would be researched as an example based on field measurement and Ecotect software simulation, and the characteristics and existing problems of natural lighting in silt houses were studied. The transformation strategies of the indoor natural lighting in silt houses are designed to solve the problem. The differenttransformation strategies aresimulated and analyzed using the software.Choosing the besttransformation strategies of the indoor natural lighting in silt houses by comparing and comprehensive evaluating. The study provides certain referencefor the improvement of the indoor light environment in the traditional silt houses.

\section{Introduction}

Stilt houses are widely distributed in southwestern region of Chinawhich is an ancient architectural form. Commonly upright stakes are used to do undercarriage. The building area is smaller, and divided into upper and lower layers. The upper living and the lower overhead for livestock or piled debris. Due to its unique architectural form, the stilt house not only used to circumvent worm beast, it also beneficial for moisture proof and ventilation. It is expression of the local population's wisdom to respond to the climatic conditions ${ }^{[1][2]}$. Because of the differences of the society and culture,stilt houses are still the most important traditions houses for ethnic minorities in Yunnan southern areas.But with the people's quality of life increasing,traditional houses cannot satisfy the people's demand for indoorenvironment.The residents have started to reconstruction the traditional houses by themselves.Many houses' characteristics have changed because lacking of a reasonable guide,and even many villages have been abandoned the Stilt houses, began to use "Chinese-style" cottage architectural forms ${ }^{[3]}$.So we must use the reasonable and appropriate transformation program to ensure the traditional houses survive.

Domestic and foreignconstruction of natural daylight study pursues on architectural lighting fashion and the overall architecture combine ${ }^{[4]}$. In order to achieve better indoor light environment by defined the building lighting mouth toward, height, size and other factors in different climatic zones.Natural lighting reconstruction must protect the style characteristic of the Wa ethnicity houses.In this paper, we through on-site measurements and softwaresimulation to study rehabilitation programs of natural daylight in stilt houses.I expected to provide some basis reference and conventional for indoor natural light transformation in stilt houses in future.

\section{2 .Evaluation criteria of natural lighting}

Research shows that residential interior natural lighting is extremely important for people's lives.Residential interior natural lighting qualityand comfort will have a direct impact on people's quality of life ${ }^{[5][6]}$. Good natural lighting including reasonable illuminance,appropriatedaylight factor, reasonable uniformity, appropriate brightness and reasonable color, where daylight factor, illuminance and uniformity are the most important criteria evaluating for the natural lighting. 


\section{1 illuminance and daylight factor}

Sufficient illuminance is the most important factor for peoples' normal life.The daylight factor refers to the reference point in the room, by the direct or indirect illumination received from assumptions and known to sky luminance distribution of the sky diffuse light generated with the same time in the outdoor unobstructed sky hemisphere produced a horizontal plane on sky plane the ratio of diffuse.Daylight factor $\mathrm{C}$ can accord the following formula:

$\mathrm{C}=\frac{\mathrm{E}_{\mathrm{n}}}{\mathrm{E}_{\mathrm{w}}} \times 100 \%$

Where $E_{n}$ - indoor illumination;

$\mathrm{E}_{\mathrm{w}}$ - outdoor illumination.

Daylightfactor andilluminanceare the most important criteria evaluating for the natural lighting.According to "Standard for Daylighting Design of Buildings" (GB50033-2013) regulations ${ }^{[7]}$,the daylight factor not less than $2.0 \%$ in residential building bedrooms and livingroom, the natural illumination not less than 300lux. Lighting standard should not be less than the specified value in Table 1 in other areas.

Table 1Lightingstandard value ofresidential building

\begin{tabular}{c|c|c}
\hline \multirow{2}{*}{ Place } & \multicolumn{2}{|c}{ Side lighting } \\
\cline { 2 - 3 } & daylight factor (\%) & illuminance (lux) \\
\hline kitchen & 2.0 & 300 \\
\hline $\begin{array}{c}\text { Bathroom, hallway, dining } \\
\text { room, stairwell }\end{array}$ & 1.0 & 150 \\
\hline
\end{tabular}

\section{2 lighting uniformity}

Lighting uniformity is the ratio of the daylight factor between the average and the lowest. Lighting uniformity is one of the important criteria for evaluating lighting. Uneven lighting illumination will make our eyes fatigue, reduce visual function and affect people's normal living. Typically, the minimum value of room lighting coefficient , or the average illumination there to have a certain limit to order to achieve better uniformity. According to "Standard for Daylighting Design of Buildings" (GB 50033-2013) provision of residential buildings daylight factor should not be higher than $7 \%$, lighting uniformity not less than 0.7 .

This paper will use lighting illumination, daylight factor and lighting uniformity to comprehensive evaluation light environment in Stilt houses.

\section{3 .The case of natural lighting simulation study in stilt houses}

\subsection{Architectural Overview}

The aerial height of the first floor is $1.8 \mathrm{~m}$ and the two floor building's exterior height is 1.4 in this paper, the research building is located in CangyuanCounty in Yunnan Provincewhich is square and the size is $8.1 \times 5.45 \mathrm{~m}$. The Building is $5.6 \mathrm{~m}$ height. The aerial height of the first floor is $1.8 \mathrm{~m}$ and the two floor building's exterior height is $1.4 \mathrm{~m}$. There are two no glass window on the top of the roof in the north and south sides, the size is $600 \times 450 \mathrm{~mm}$. Thatch and woodare mainlymaterials, its reflectance is 0.3. Houses appearance shown in Figure 1.

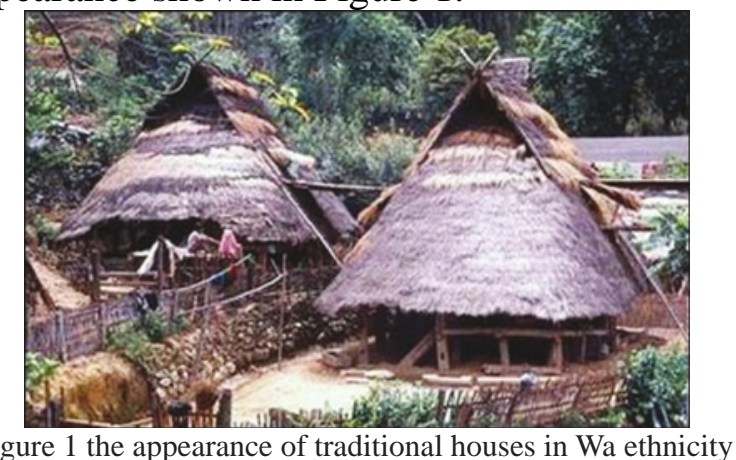

\subsection{Natural lighting simulation in residential}

Ecotect use basic model to analysis lighting in full cloudy. The full cloudy is the most adverse conditions for lighting.It is very suitable for building to comprehensive lighting evaluation, 
therefore, and this paper will use Ecotect2011 software to simulate natural light.

Press on the "China Light Climate partition" standard, Cangyuan County is located in Class II light climate partition, its natural light design illuminance take 16500lux in outdoor. We will use International CommissionModel in the full cloudy and calculate the most adverse conditions of the lighting. Take $0.75 \mathrm{~m}$ high from the ground level as the reference plane, grid nodes is 480 .The simulation results of lighting are shown in figures 2 and 3.

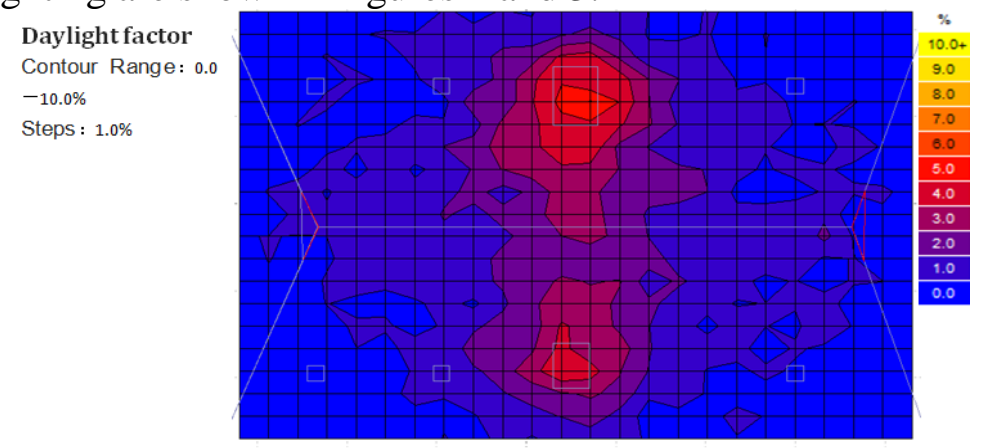

Figures 2Thesimulation results of daylight factor

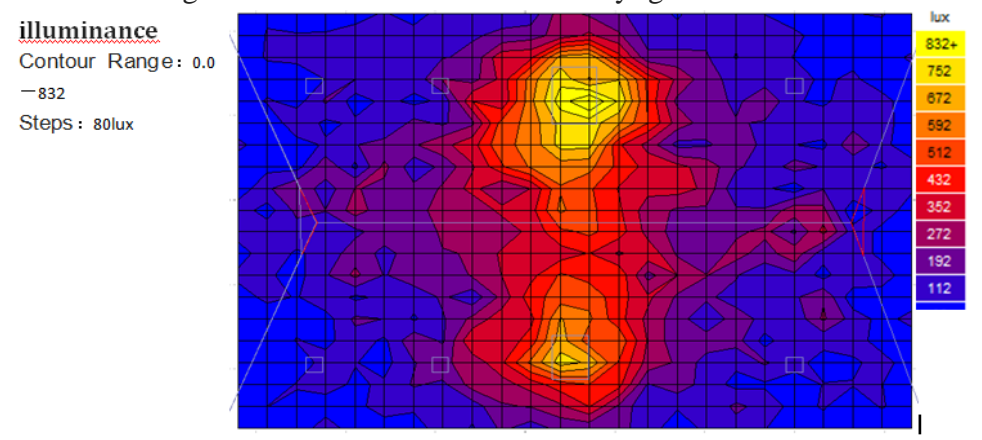

Figures 3 Thesimulation results of illuminance

\subsection{Environment lighting measured values in Residential}

To layout the measuring points in throughout the room.There is a measuring point from each $2 \mathrm{~m}$ in laterally and each $1 \mathrm{~m}$ in longitudinal. Take $0.75 \mathrm{~m}$ high from the ground level as the reference plane. There are nine measuring points in the room. Figure 4 is the distribution of measuring point.

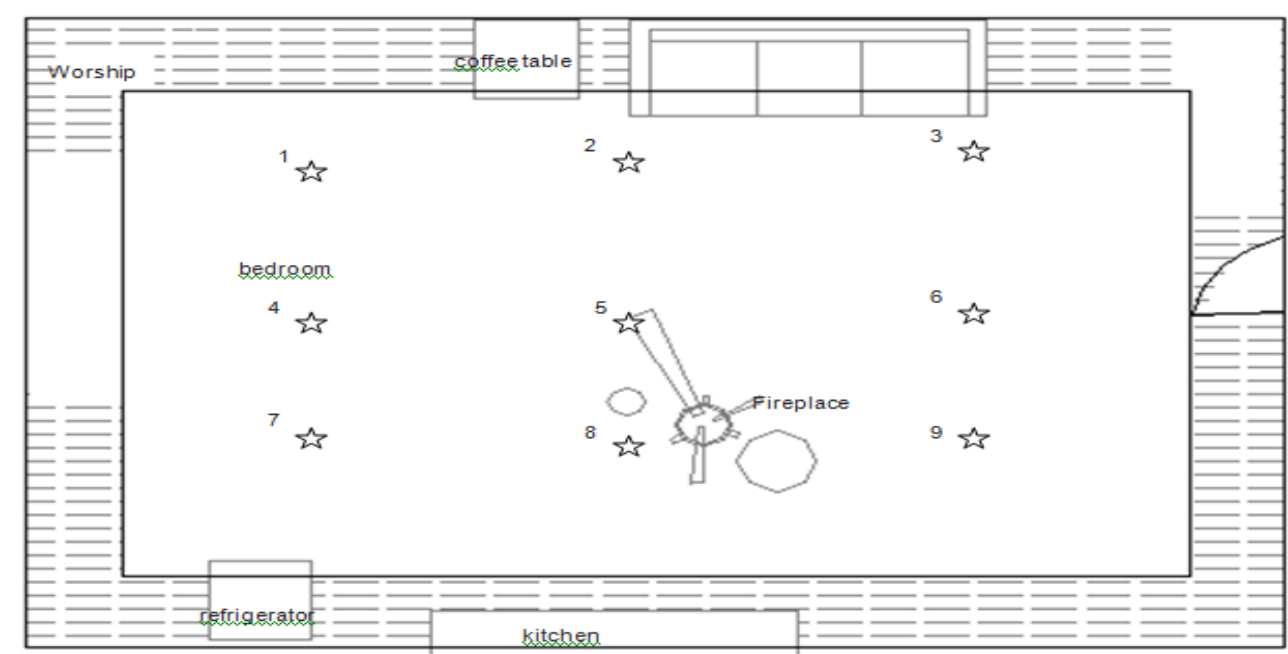

Figure 4 the distribution of measuring point

The lightingweremeasured when the weather is the full cloudy at 10 o'clock on April 23, 2014. Each measuring point lighting weremeasured and the outdoor illumination were measured at the same time. The measured valuesof the environment lighting are shown in table 2. 
Table 2 the measured values of the environment lighting

\begin{tabular}{c|c|c|c|c|c}
\hline \multirow{2}{*}{$\begin{array}{c}\text { Measuringpoint } \\
\text { number }\end{array}$} & $\begin{array}{c}\text { Indoor } \\
\text { illumination } \\
\text { (lux) }\end{array}$ & $\begin{array}{c}\text { Measured values } \\
\text { illumination } \\
\text { (lux })\end{array}$ & $\begin{array}{c}\text { daylight factor } \\
(\%)\end{array}$ & $\begin{array}{c}\text { Simulation } \\
\text { ofdaylight } \\
\text { factor }(\%)\end{array}$ & $\begin{array}{c}\text { deviation } \\
(\%)\end{array}$ \\
\hline 1 & 120 & 14318 & 0.84 & 0.89 & -5.95 \\
\hline 2 & 493 & 14425 & 3.42 & 3.64 & -6.43 \\
\hline 3 & 136 & 14482 & 0.95 & 0.89 & 6.32 \\
\hline 4 & 99 & 14519 & 0.68 & 0.72 & -5.88 \\
\hline 5 & 426 & 14436 & 2.95 & 2.79 & 5.42 \\
\hline 6 & 54 & 14534 & 0.37 & 0.35 & 5.41 \\
\hline 7 & 88 & 14621 & 0.6 & 0.63 & -5.00 \\
\hline 9 & 490 & 14659 & 3.34 & 3.58 & -7.19 \\
\hline
\end{tabular}

The result can be found by contrastedmeasured values and simulation values that they have less difference. The maximum deviation is $7.19 \%$ at the measuring point 8 . By the measured values, we can know the simulation values of lighting are right.

\subsection{Analysis}

The simulation values of daylight factor and lighting illuminancein house was shown in Table 3.

Table 3 the simulation values of daylight factor and lighting illuminance

\begin{tabular}{c|c|c|c}
\hline $\begin{array}{c}\text { Daylight factorrange } \\
(\%)\end{array}$ & illuminance (lux) & $\begin{array}{c}\text { Ratio ofwithinrange } \\
(\%)\end{array}$ & $\begin{array}{c}\text { Ratioof the under the } \\
\text { range (\%) }\end{array}$ \\
\hline $0 \sim 1$ & $0 \sim 165$ & 73.46 & 100 \\
\hline $1 \sim 2$ & $165 \sim 330$ & 12.33 & 26.54 \\
\hline $2 \sim 3$ & $330 \sim 495$ & 11.16 & 3.21 \\
\hline $3 \sim 4$ & $495 \sim 660$ & 2.74 & 0.31 \\
\hline $4 \sim 5$ & $660 \sim 825$ & 0.31 & 0.31 \\
\hline
\end{tabular}

The average daylight factor is $1.4 \%$, the average illumination is 231lux and the lighting uniformity is 0.18.It is very bad lighting for the building. There is just $14.21 \%$ areas' daylight factor and illumination in the room can meet the requirements of "Standard for daylighting Design of Buildings" (GB50033-2013) regulations.

Throughresearching the stilt houses, there are two main reasons to cause the poor lighting conditions can be found. First, the window area is too small in the building. The small window makes the badillumination and poorlighting uniformity. The second is the mainlymaterials of the stilt houses are thatch and wood that have very low reflectivity. Therefore, it is necessary to take measuresto improve and upgrade the light environment in the stilt houses.

\section{4 .The scheme of improving the lighting environment in stilt houses}

\subsection{Improving program design}

Since the smaller area of the window, there is a poor quality oflightingenvironment in the house, so we should increase the lighting port area to meet the needs of daily life with natural daylight. It should be kept in the structural characteristics of the building's original premise at the same time.In this paper we will present three simple plans to improvement the lighting environment:

1.Expand the port area of lighting, expansion the top of the roof window area from $600 \times 450 \mathrm{~mm}$ to $1200 \times 600 \mathrm{~mm}$ in direct.

2.The top of the roof window will be abandoned. There are four no glass window on the top of the roof in the north and south sides, the size is $900 \times 650 \mathrm{~mm}$.

3. There are four side window will be increased in the north and southfacades, the size of $1000 \times$ $600 \mathrm{~mm}$.

\subsection{The simulation results ofthe improvementplan}

The design value ofthe lightingilluminanceand the design parameters of building architectural will be unchanged. Take $0.75 \mathrm{~m}$ high from the ground level as the reference plane.The results of natural lighting simulation are shown in figures 5 to 7. 


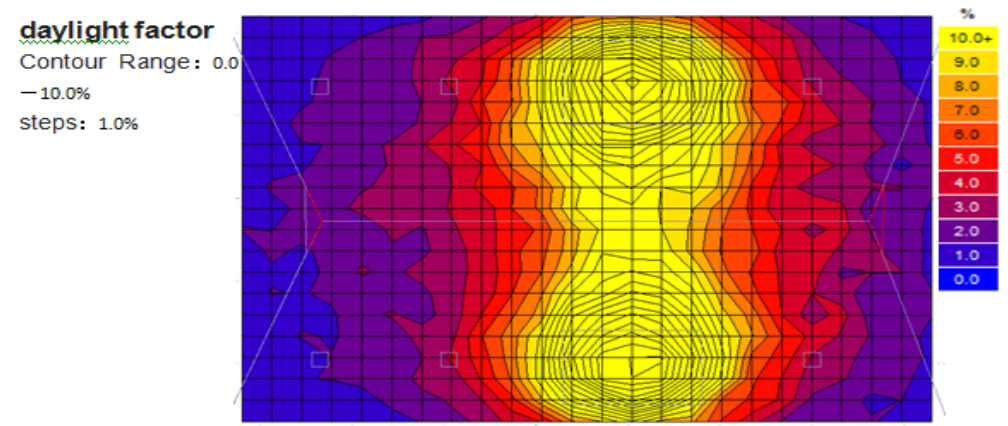

Figures 5 the results of daylightfactorsimulation for plan 1

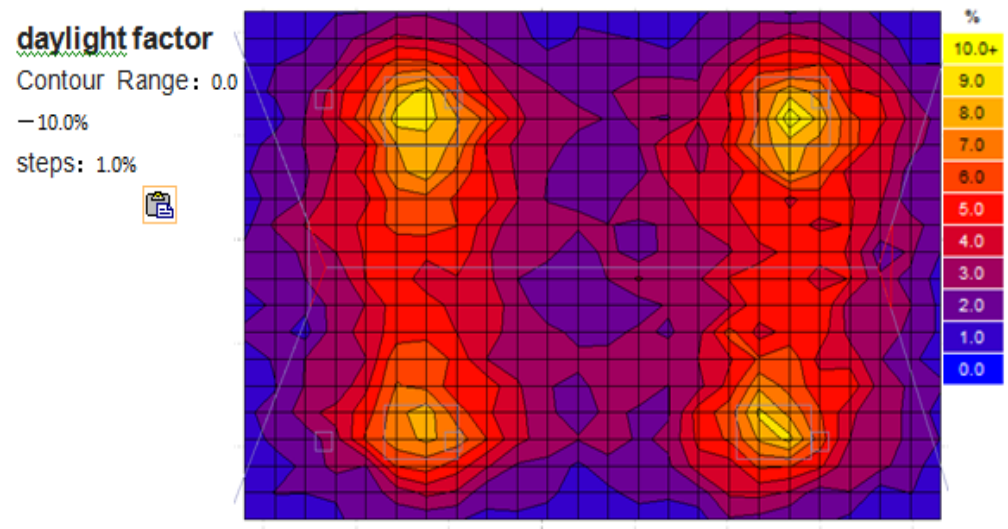

Figures 6 the results of daylightfactorsimulation for plan 2

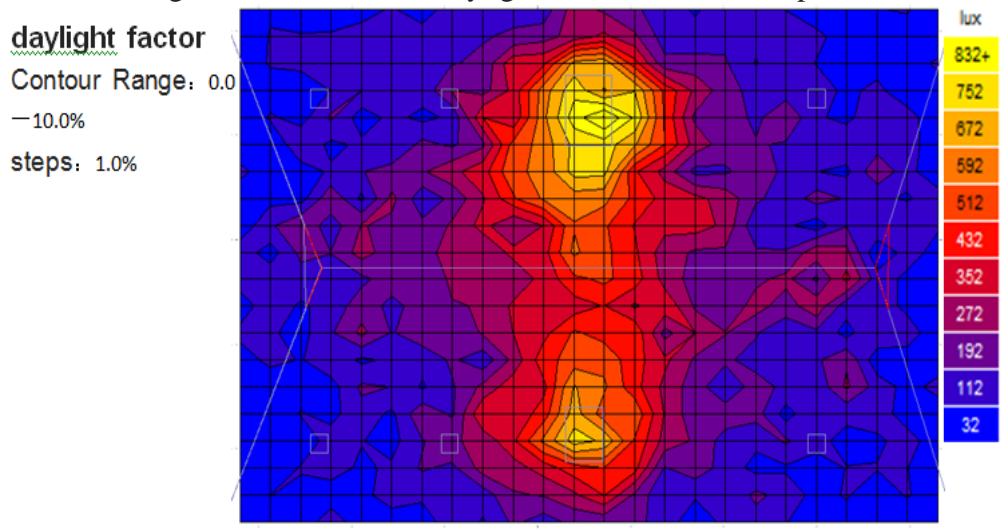

Figures 7 the results of daylightfactorsimulation for plan 3

\subsection{Comparison of improvement plan}

The different lighting effects of improvementplanare shown in Table 4 by calculating.

Table4the differentlighting effectsofimprovementplan

\begin{tabular}{c|c|c|c|c}
\hline plan & $\begin{array}{c}\text { The average } \\
\text { daylight factor } \\
(\%)\end{array}$ & $\begin{array}{c}\text { The average } \\
\text { illuminance } \\
\text { (lux) }\end{array}$ & $\begin{array}{c}\text { Lighting } \\
\text { uniformity }\end{array}$ & $\begin{array}{c}\text { Ratio of meet the } \\
\text { standards (\%) }\end{array}$ \\
\hline Original building & 1.4 & 231 & 0.18 & 14.21 \\
\hline 1 & 3.28 & 541 & 0.19 & 60.4 \\
\hline 2 & 3.47 & 573 & 0.55 & 95.7 \\
\hline 3 & 1.99 & 328 & 0.33 & 35.4 \\
\hline
\end{tabular}

We can see that the lighting illumination have improved after the transformation through the simulation analysis.In option one, the average daylight factor is 3.28\% throughincrease the window areain direct. The average illuminance is 541lux.But we can find through the simulation that the lightingimprovement can only change the area where near the mouth of the window. The Lighting uniformity is only 0.18 . There is only $60.4 \%$ area meet therequirements of"Standard for lighting Design of Buildings" (GB50033-2013) regulations.In option two, the average daylight factor is $3.47 \%$ throughincrease the number of window. The average illuminance is 573lux. The Lighting uniformity is 0.55 . There is $95.57 \%$ area meet therequirements of"Standard for daylighting Design of Buildings" (GB50033-2013) regulations.In option three, the average daylight factor is1.99\% 
throughincrease the side window. So the side windows are not suitable fornatural lighting in stilt houses $^{[8]}$.

The option twois the best way for the natural lighting transform in the stilt houses although three schemes are unable to meet the requirements of the lighting uniformity which not less than 0.7. Moreover, we should choice the materials which surface is smooth, easy to clean and have high reflectivity to improve light intensity and lighting uniformity.

\section{5 .Conclusion}

We have found the better way to improve natural lighting for Waethnicity' stilt houses throughthe measured and simulated analysis.And we can know the following conclusions:

(1)Computer simulation is a feasible method for natural lighting traditional in stilt houses. We can use numerical simulation to find the most reasonable rehabilitation programs for the natural lighting traditional.

(2)The main reason for the poor quality of lighting is small area of architectural lighting mouth in the stilt houses, so we should improve the quality of natural light by increased the main port area.

(3)Skylights arebetter choice for improvingthe quality of natural light than side window in Waethnicity, becauseof the characteristic of the houses.

(4)If we expansion the window area in direct, the lightingimprovement can only change the area where near the mouth of the window,the lighting uniformity is bad. So we should not expansion the window area in direct in stilt houses.

\section{Acknowledgements}

This work was financially supportedby:

(1)National Key Technology R\&D Program of the Ministry of Science and Technology, under Grant No.2013BAJ07B01.

(2) National Natural ScienceFoundation of China, under Grant No.51268020.

\section{Reference}

[1]Zhao Hairu. The architecture culture and shape characteristics of Stilt houses in WaNationality[J], Scientific Consult, 2014,(1):81.

[2]Liu Yuxian. A comparative study on Pile-Dwelling of Dai Nationality and other Nationalities in Yunnan province[D],Kunming University of Science and Technology,2010.

[3]Zhou Huike. The Research of Technology Strategy during the Updating of Climate-Dominated Stilt Houses in Southern Yunnan Province-Taking Langcang County in Jingmai Area as an Example[D],Kunming University of Science and Technology,2013.

[4]Yanwei. To discuss Design optimization of building Natural lighting[D],TongjiUniversity,2009.

[5]P.G.Hopkinson,Petherbridge,J.Longmore.Daylight,The IES Code:Recommendations for good interior fighting 1966.

[6]Richard Smith.Light and health: A broad overview 1986(2).

[7]GB 50033-2013,Standard for daylighting design of buildings. Beijing: Chinaarchitecture \&Building press.

[8]Zhangcheng,WangDongmei,YangMochi.Analysis on the Influences of External Shading on Building Energy Consumption and Daylighting Effect in Hot-summer and Cold-winter Region[J], Building Science,2011,27(4). 